\title{
Quality Evaluation of Nile Tilapia Fish (Oreochromis niloticus) Fillets by Using Chitosan and Nanochitosan Coating during Refrigerated Storage
}

\author{
Walaa Mohamed Elkassas ${ }^{1 *}$, Shereen Abdelfattah $\operatorname{Yassin}^{1}$ and Mohamed Nashat Saleh ${ }^{2}$ \\ ${ }^{1}$ Department of Food Hygiene, Animal Health Research Institute, Kafrelsheikh Lab, Agriculture Research Center (ARC), Egypt. \\ ${ }^{2}$ Food Technology Research Institute, Kafrelsheikh Lab, Agriculture Research Center (ARC), Egypt. \\ *Corresponding author`s Email: w.elkasas77@yahoo.com; (DORCiD: 0000-0001-5077-1223
}

\begin{abstract}
Using natural preservatives has a probability to improve the quality and integrity of fish products. Such research investigated the antimicrobial and antioxidant effects of chitosan and chitosan nanoparticles casing on the quality of tilapia (Oreochromis niloticus) fish fillets through refrigerated storage. In the present investigation solutions of chitosan (1 and 2\%) and nanochitosan (1 and 2\%) were applied for the casing of tilapia fish slices thereafter stored at $4^{\circ} \mathrm{C}$ for 15 days. Uncoated (control) and coated fish fillets pieces were examined intermittently for bacteriological parameters (Total bacterial count, Proteolytic bacterial count, Lipolytic bacterial count, and Staphylococcus aureus count), quality parameters ( $\mathrm{pH}$, total volatile basic nitrogen (TVB-N), and thiobarbituric acid reactive substances, TBARS) and sensory features. Results showed that $2 \%$ chitosan and $2 \%$ chitosan nanoparticle solutions were the optimal concentrations for improving the quality of tilapia fish fillets until 10 days of refrigerated storage period compared to the control group. However, using $2 \%$ chitosan nanoparticles showed higher antimicrobial activity, strong ability in preventing protein degradation, retarding lipid oxidation, accepted $\mathrm{pH}$ values and delay in declining of sensory score more than $2 \%$ chitosan solution during the storage period. Therefore, $2 \%$ chitosan nanoparticles as a natural preservative can be utilized for the conservation of quality properties and expanding the shelf life of tilapia fish slices through chilled storage.
\end{abstract}

Key words: Bacteriological and quality parameters, Chitosan, Nanochitosan, Tilapia fish fillets

\section{INTRODUCTION}

Fish products are highly susceptible to quality deterioration, probably due to lipid oxidative reactions, in particular PUFAs. Such reactions are stimulated (catalyzed) by the presence of high heme and nonheme protein concentrations. These proteins are known to contain iron and other metal ions in their structures (Decker and Haultin, 1992). In addition, the quality of seafood is strongly affected by autolysis, bacterial contamination and loss of protein functionality (Jeon et al., 2002). Tilapia (Oreochromis niloticus) is a freshwater fish species that has been commonly cultured worldwide and sold in general stores and food market chains, but its preservation has been a problem for a long time due to its brief shelf life. Hence, effective methods to extend the shelf-life of tilapia need to be created. In order to improve the microbial quality and increase the shelf-life of seafood products, food preservation methods like freezing, chemical preservation, salting, and modified atmosphere packaging were utilized. In spite of the simple and widespread use of preservatives, both food processors and consumers have wanted to reduce the use of synthetic chemicals to preserve foods. As a result, interest in the application of natural agents as bio-preservatives has been growing, while most natural agents have low antimicrobial activity spectrums and only effect in very high concentrations. Chitosan shows antimicrobial action against a wide range of foodborne microorganisms, thereby gaining attention as a possible natural preservative for food (Raafat and Sahl, 2009; Friedman and Juneja, 2010). Chitosan, a linear polysaccharide of randomly distributed $\beta$-(1-4)-linked D-glucosamine and N-acetyl-D-glucosamine is a biocompatible polysaccharide gotten from chitin deacetylation that is commonly found in nature, such as in shrimps, crabs and fungi. Chitosan coatings have been widely used in the food industry due to certain benefits such as edibility, biodegradability, cosmetic appearance and barrier properties, being non-toxic and non-polluting, as well as being a carrier of food additives (i.e., antioxidants, antimicrobials). So, by preventing bacterial growth and delaying lipid oxidation, these coatings can maintain the quality of raw, frozen, and processed foods including fish products. Chitosan antimicrobial action has been illustrated against many bacteria, fungi and yeasts, possessing a high killing rate against Gram-positive and Gram-negative microbes but low poisonous towards mammalian cells (Kong et al., 2010). The antimicrobial action mechanism of chitosan has not yet been completely elucidated but several theories have been suggested. Due to interactions between the positively charged chitosan molecules and bacterial cell membrane charged negative, the most plausible explanation is a shift in cell 
permeability. This interaction results in the leakage of protein and other intracellular components (Papineau et al., 1991; Fang et al., 1994). Other techniques are the interaction of diffused hydrolysis products with microbial DNA, resulting in mRNA and protein synthesis inhibition (Sudarshan et al., 1992) and the chelation of metal, spore components and fundamental nutrients (Cuero et al., 1991). Nanoparticles are prepared from natural or artificial polymers with the order of 100 nanometers $(\mathrm{nm})$ or less in one or more dimensions (Sinha and Okamoto, 2003). Nanoparticles have demonstrated unusual physical and chemical characteristics due to special effects such as the quantum size, small size, surface, and macro quantum tunnel effects. Due to the advantages of chitosan nanoparticle over other conventional materials, their use as food packaging materials has increased recently (Ramezani et al., 2015). In addition, chitosan nanoparticles have inhibited bacterial growth in food because of the antimicrobial properties (Du et al., 2009). Furthermore, using nanoparticles of chitosan-tripolyphosphates retained antioxidant activity in vitro using free radical scavenging and reducing power tests (Zhang et al., 2008). Therefore, it is beneficial to produce natural preservative coatings or films with antioxidant and antibacterial activities that increase the shelf life of fish and fish products. Hence, the object of this research was to investigate the antimicrobial and antioxidant effects of chitosan and chitosan nanoparticles coatings on the quality of chilled $\left(4 \pm 1^{\circ} \mathrm{C}\right)$ Tilapia fish fillets.

\section{MATERIALS AND METHODS}

\section{Ethical approval}

The current study was approved by the Ethical Committee for life fish sampling at the Animal Health Research Institute, Agriculture Research Center (ARC), Egypt (License No. AHRI, 184429).

\section{Preparation of chitosan and chitosan nanoparticles}

Chitosan solution was made by dissolving $1 \%$ (w/v) chitosan (Meron Chemical Co., low molecular weight, moisture $10 \%$ max., Marine Chemicals, India) in $1 \%(\mathrm{v} / \mathrm{v})$ acetic acid. To realize the total scattering of chitosan, the solution was blended using a magnetic stirrer at room temperature $\left(25^{\circ} \mathrm{C}\right)$ for melting totally. Glycerol was added up to $0.75 \mathrm{~mL} / \mathrm{g}$ as a plasticizer and blended for $10 \mathrm{~min} .2 \%$ chitosan was also prepared in the same way. Nanoparticles were attended by cross-linking of chitosan-sodium tripolyphosphate solution (Ch-TPP). Chitosan (1\%) was melted in $1 \%$ acetic acid. Sodium tripolyphosphate solution $(1 \%, \mathrm{w} / \mathrm{v})$ was melted in distilled water. By magnetic stirring at room temperature (25$30{ }^{\circ} \mathrm{C}$ ), $4 \mathrm{~mL}$ of sodium tripolyphosphate solution was included in $100 \mathrm{~mL}$ of chitosan solution. The blend was mixed for $60 \mathrm{~min}$, at that point, treated with sonication (Model 300VT, $115 \mathrm{~V}, 60 \mathrm{~Hz}$, Manassas, VA, USA) at $1.5 \mathrm{~kW}$ for $10 \mathrm{~min}$, sometime recently being utilized for further examination and also, $2 \%$ nanochitosan was prepared by the same way (Du et al., 2009). Figure 1 showed that the average particle size $(\mathrm{nm})$ of the Ch-TPP nanoparticle was measured using a Transmission electron microscope (TEM) of 2000 kV (Jem-100SX model, Japan) in the Faculty of Medicine, Tanta University. The average particle size $(\mathrm{nm})$ of Ch-TPP nanoparticle was $100 \mathrm{~nm}$.

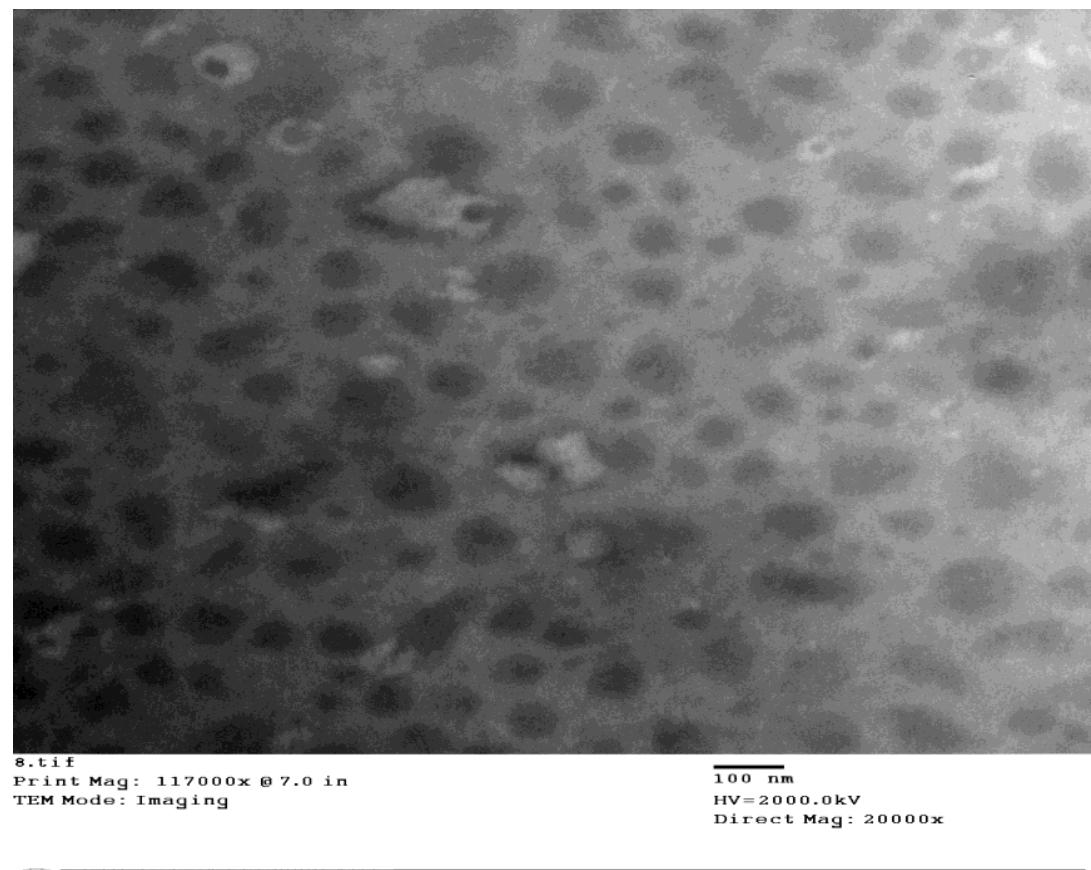

Figure 1. Transmission electron microscope of high voltage (2000 KV) exposed to the average particle size (100 nm) of the Ch-TPP nanoparticle with direct magnification $(20000 \mathrm{x})$ and the image of 8.tif (tagged image file format) with print magnification of 117000x@7.0. 


\section{Sample preparation}

Thirty tilapia fish (Oreochromis niloticus) with mean weight of 450-500 g were bought from a regional fish shop in Kafrelsheikh town. The fish were freshly hunted and were kept in ice with a fish/ice proportion of 1:2 (w/w) and transported to the laboratory inside $1 \mathrm{~h}$. Upon entry, the fish were washed in cool faucet water then every fish was accurately filleted by hand. Two skins on fillets were gained from every fish after taking off the head and bone. 60 slices of fish (fillet: $5 \mathrm{~cm} \times 10 \mathrm{~cm}$ ) were partitioned randomly into five treating sets (12 fillets in each set) and were given a dip treatment in $1 \%$ acetic acid (control), $1 \%$ chitosan, $2 \%$ chitosan, $1 \%$ nanochitosan, and $2 \%$ nanochitosan solution up to $20 \mathrm{~min}$. At that point, the fillets were taken out and permitted to deplete for $2 \mathrm{~h}$ at $20^{\circ} \mathrm{C}$ on a pre-sterilized metal net to make the edible casing, at that point stocked at $4^{\circ} \mathrm{C}$ for the following quality evaluation (Alboghbeish and Khodanazary, 2018). Sensorial, Physicochemical, and microbiological examinations were carried out at 5-days interims up to 15 days to confirm the total goodness of fish.

\section{Sensory analysis}

The total acceptability of tilapia fish slices was confirmed by a five-point measure taking into consideration texture, color, and smell. Specialists (6-member trained panel) were recorded for sensory features, such as color discoloration (score 5 means no discoloration; till score 1: which means extraordinary discoloration); smell (score 5: means amazingly desirable; till score 1: refers to greatly unacceptable/off-odors), and texture (score 5: means firm; and score 1: refers to extremely smooth). The mediums of these scores were described as total acceptability (Score 5: greatly desirable; 4: good; 3: average; 2: questionable and finally score 1: greatly inadmissible). Shelf life standards supposed that repudiation would happen when the sensory traits declined underneath 4.0 (Ojagh et al., 2010).

\section{Physicochemical examination}

\section{Measurement of $\mathrm{pH}$}

The $\mathrm{pH}$ rate was measured by utilizing an electrical pH meter (Bye model 6020, USA) according to Pearson (2006).

\section{Measurement of total volatile basic nitrogen}

TVB-N of Oreochromis niloticus fish fillets was measured as stated in ES: 63-9/ (2006).

\section{Measurement of thiobarbituric acid reactive substances}

This test depends on determination of malonaldehyde (MDA) as an end product of lipid peroxidation and was done according to ES: 63-10/ (2006).

\section{Microbiological analysis}

10 grams of fish meat was carried aseptically to a stomacher bag including $90 \mathrm{~mL}$ of $0.1 \%$ peptone water. Fish flesh was homogenized for $60 \mathrm{~s}$ using a stomacher beneath sterilized conditions, to obtain 1/10 dilution. Serial dilutions were getting ready to be utilized for enumeration of total bacterial count (TBC), proteolytic (PBC), Lipolytic (LBC), and Staphylococcus aureus count. TBC was cultivated on standard plate count agar, PBC (on skim milk agar), LBC (on butterfat agar) and Staphylococcus aureus (on Baird Parker agar). Fish fillets bacterial counts were confirmed as stated in APHA (2002). The TBC was incubated at $37^{\circ} \mathrm{C} / 48 \mathrm{hr}$; PBC, and $\mathrm{LBC}$ at $30^{\circ} \mathrm{C}$ for $48 \mathrm{hr}$ and $37^{\circ} \mathrm{C}$ for $48 \mathrm{hr}$ for Staphylococcus aureus count. The bacterial colonies were counted as CFU/g.

\section{Statistical analysis}

All estimations were reproduced three times to every set and average values \pm standard errors were registered for each case. Analysis of variance (ANOVA) was done and average comparisons were achieved by Duncan's multiple range tests utilizing SPSS (Statistical Package for the Social Sciences) to evaluate the importance of differences among average values. $\mathrm{P}$ values lower than 0.05 were deemed statistically significant.

\section{RESULTS}

\section{Sensory analysis}

The findings of the sensory assessment of fish fillets are shown in table 1. During the first days of storing period, no significant differences have been identified between the control sensory scores and other treatments where all the score values were $4.97 \pm 0.03(\mathrm{P}<0.05)$. On the fifth day, the control samples displayed an observed decrease in the freshness score $(2.83 \pm 0.17)$ which became unacceptable, while at fifth day, $1 \%$ chitosan and $1 \%$ nanochitosan freshness score were $4.17 \pm 0.17$; however, $2 \%$ chitosan and2\%nanochitosan were $4.67 \pm 0.17$. Until day tenth, it observed that $2 \%$ chitosan and $2 \%$ nanochitosan treated samples had significantly $(\mathrm{P}<0.05)$ higher scores $(4.1 \pm 0.21$ and $4.33 \pm 0.17)$ in the overall acceptability than the other treatment groups $(1.5 \pm 0.29,2.83 \pm 0.4$ and $3 \pm 0.29$ in control, $1 \%$ chitosan and $1 \%$ nanochitosan, respectively). 
Table 1. Statistical analysis results of overall acceptability values of fish fillet samples

\begin{tabular}{lccccc}
\hline Groups & Control & $\begin{array}{c}\text { Chitosan } \\
(\mathbf{1 g ~ C h} / \mathbf{1 0 0} \mathbf{~ D W})\end{array}$ & $\begin{array}{c}\text { Chitosan } \\
(\mathbf{2 g ~ C h} / \mathbf{1 0 0 m l} \mathbf{D W})\end{array}$ & $\begin{array}{c}\text { Nanochitosan } \\
(\mathbf{1 g ~ N C h} / \mathbf{1 0 0 m l} \mathbf{D W})\end{array}$ & $\begin{array}{c}\text { Nanochitosan } \\
(\mathbf{2 g} \text { NCh/100ml DW })\end{array}$ \\
\hline First day & $4.97 \pm 0.03^{\mathrm{a}}$ & $4.97 \pm 0.03^{\mathrm{a}}$ & $4.97 \pm 0.03^{\mathrm{a}}$ & $4.97 \pm 0.03^{\mathrm{a}}$ & $4.97 \pm 0.03^{\mathrm{a}}$ \\
$5^{\text {th }}$ day & $2.83 \pm 0.17^{\mathrm{b}}$ & $4.17 \pm 0.17^{\mathrm{a}}$ & $4.67 \pm 0.17^{\mathrm{a}}$ & $4.17 \pm 0.17^{\mathrm{a}}$ & $4.67 \pm 0.17^{\mathrm{a}}$ \\
$10^{\text {th }}$ day & $1.5 \pm 0.29^{\mathrm{c}}$ & $2.83 \pm 0.4^{\mathrm{b}}$ & $4.1 \pm 0.21^{\mathrm{a}}$ & $3.0 \pm 0.29^{\mathrm{b}}$ & $4.33 \pm 0.17^{\mathrm{a}}$ \\
$15^{\text {th }}$ day & $1.17 \pm 0.17^{\mathrm{c}}$ & $2.17 \pm 0.17^{\mathrm{b}}$ & $3.17 \pm 0.3^{\mathrm{a}}$ & $2.33 \pm 0.17^{\mathrm{b}}$ & $3.67 \pm 0.17^{\mathrm{a}}$ \\
\hline
\end{tabular}

Means of different superscript letters within the same row differ significantly inP< 0.05 . Ch: Chitosan, NCh: Nanochitosan, DW: Distilled water

\section{Physicochemical examination \\ $p H$}

During refrigerated storing, changes in $\mathrm{pH}$ values were seen in table 2 . The first $\mathrm{pH}$ values of all fish specimens was $6.18 \pm 0.15,6.18 \pm 0.16,6.09 \pm 0.12,6.13 \pm 0.01$, and $5.96 \pm 0.07$ in control, $1 \%$ chitosan, $2 \%$ chitosan, $1 \%$ nanochitosan, and $2 \%$ nanochitosan, respectively, and increased to $6.72 \pm 0.26,6.58 \pm 0.02,6.29 \pm 0.01,6.39 \pm 0.02$, and $6.21 \pm 0.01$ at fifth day, respectively. At tenth day, $\mathrm{pH}$ values were $8.22 \pm 0.05,6.87 \pm 0.05,6.30 \pm 0.02,6.46 \pm 0.02$ and $6.24 \pm 0.03$, respectively until reached to $8.63 \pm 0.06,6.88 \pm 0.04,6.48 \pm 0.02,6.62 \pm 0.02$, and $6.30 \pm 0.02$, respectively at fifteenth day of refrigerated storage.

\section{Total volatile basic nitrogen}

Table 3 showed the changes in Total volatile basic nitrogen (TVB-N) values of fish samples during refrigerated storing. The values of TVB-N initially were $6.18 \pm 1.24,6.03 \pm 1.18,5.04 \pm 0.83,6.04 \pm 1.22$, and $4.89 \pm 0.73 \mathrm{mg} / 100 \mathrm{~g}$ of fish in control, $1 \%$ chitosan, $2 \%$ chitosan, $1 \%$ nanochitosan, and $2 \%$ nanochitosan respectively. TVB-N rates of Tilapia fillets gradually increased in all treatments with storing time. At fifth day TVB-N values were $36.73 \pm 0.26,22.03 \pm 0.1$, $16.73 \pm 0.34,19.15 \pm 0.13$, and $14.01 \pm 1.1 \mathrm{mg} / 100 \mathrm{~g}$ of fish, respectively. TVB-N values recorded $47.88 \pm 0.79,29.99 \pm 0.63$, $20.68 \pm 0.9,27.04 \pm 1.32$, and $17.95 \pm 0.53 \mathrm{mg} / 100 \mathrm{~g}$ of fish at tenth day, respectively. Finally, at the fifteenth day of storing period, samples coated with chitosan and nanochitosan had markedly lower TVB-N values $(\mathrm{P}<0.05)$ than control samples $(54.62 \pm 0.27 \mathrm{mg} / 100 \mathrm{~g}$ of fish). Mean of TVB-N values of $2 \%$ chitosan and $2 \%$ nanochitosan were $22.93 \pm 0.55$ and $19.75 \pm 0.93 \mathrm{mg} / 100 \mathrm{~g}$ of fish, respectively on day fifteenth of storing.

\section{Thiobarbituric acid reactive substances}

The initial values of Thiobarbituric acid (TBA) reactive substances in table 4 was $1.26 \pm 0.45,1.12 \pm 0.40,0.67 \pm 0.19$, $0.90 \pm 0.04$ and $0.56 \pm 0.15 \mathrm{mg} \mathrm{MDA} / \mathrm{kg}$ of fish in untreated (control), $1 \%$ chitosan, $2 \%$ chitosan, $1 \%$ nanochitosan and $2 \%$ nanochitosan group, respectively. Values of TBA in the control, chitosan, and nanochitosan coating samples increased with storing time. At fifth day, TBA values reached $4.83 \pm 0.06,3.89 \pm 0.07,2.24 \pm 0.04,3.23 \pm 0.29$, and $1.18 \pm 0.24 \mathrm{mg}$ $\mathrm{MDA} / \mathrm{kg}$ of fish, respectively. On day 10 , samples coated with $1 \%$ and $2 \%$ chitosan $(4.69 \pm 0.05,3.55 \pm 0.20)$ and $1 \%$ and $2 \%$ nanochitosan $(4.24 \pm 0.06,3.21 \pm 0.04)$ had markedly lower TBA rates other than the control value $(5.60 \pm 0.04 \mathrm{mg}$ MDA $/ \mathrm{kg}$ of fish) $(\mathrm{P}<0.05)$. TBA mean values of $2 \%$ chitosan and 1 and $2 \%$ nanochitosan were $4.16 \pm 0.09,4.31 \pm 0.04$, and $3.95 \pm 0.05 \mathrm{mg} \mathrm{MDA} / \mathrm{kg}$ of fish, on day fifteenth of storing time, respectively. While, control and $1 \%$ chitosan were $6.87 \pm 0.03$ and $4.78 \pm 0.06 \mathrm{mg} \mathrm{MDA} / \mathrm{kg}$ of fish.

\section{Microbiological examination}

During refrigerated storing, the variation in total bacterial count (TBC) was shown in table 5. The first TBC values were $5.15 \pm 4.23,5.0 \pm 4.64,4.18 \pm 3.54,5.04 \pm 4.58$ and $3.97 \pm 3.36 \log 10 \mathrm{cfu} / \mathrm{g}$ in control, $1 \%$ chitosan, $2 \%$ chitosan, $1 \%$ nanochitosan and $2 \%$ nanochitosan, respectively. At fifth day TBC reached 5.92 $\pm 5.41,5.11 \pm 4.36,4.88 \pm 4.45,5.08 \pm 4.36$ and $4.95 \pm 3.46 \log 10 \mathrm{cfu} / \mathrm{g}$, respectively. Among all treatments, sample treated with $2 \%$ chitosan and $2 \%$ nanochitosan $(5.29 \pm 4.82$ and $4.99 \pm 4.51 \log 10 \mathrm{cfu} / \mathrm{g})$ had lower TBC at tenth day than control, $1 \%$ chitosan and $1 \%$ nanochitosan $(6.26 \pm 5.41,6.11 \pm 5.97$, and $6.08 \pm 5.62 \log 10 \mathrm{cfu} / \mathrm{g}$, respectively) and within the acceptable limit of Egyptian Organization for Standardization and Quality Control (EOS) (2005).

The variations in proteolytic (PBC) and lipolytic bacterial counts (LBC), respectively through the storage periods are presented in tables 6 and 7. The first values of PBC in the fish slices were 4.57 $\pm 2.95,4.41 \pm 2.95,4.08 \pm 3.18$, $4.11 \pm 4.41$, and $4.04 \pm 3.98$ and for $\mathrm{LBC}$ were $3.98 \pm 3.89,3.71 \pm 3.71,3.28 \pm 3.60,3.68 \pm 4.28$, and zero $\log 10 \mathrm{cfu} / \mathrm{g}$, in control, $1 \%$ chitosan, $2 \%$ chitosan, $1 \%$ nanochitosan and $2 \%$ nanochitosan group, respectively. During the storage period PBC and LBC values increased gradually within each treatment. At fifth day, PBC values were 5.23 $\pm 3.76,5.11 \pm 4.18$, $5.04 \pm 4.08,5.08 \pm 3.94$ and $4.72 \pm 4.32 \log 10 \mathrm{cfu} / \mathrm{g}$, while $\mathrm{LBC}$ values were $5.46 \pm 4.26,5.36 \pm 4.58,4.71 \pm 4.53,5.3 \pm 5.32$ and $4.51 \pm 4.04 \log 10 \mathrm{cfu} / \mathrm{g}$ in control, $1 \%$ chitosan, $2 \%$ chitosan, $1 \%$ nanochitosan, and $2 \%$ nanochitosan samples, respectively. At tenth day of the storing time, values of the treated sets with $2 \%$ chitosan and $2 \%$ nanochitosan $(5.85 \pm 5.28$ and $5.71 \pm 5.41 \log 10 \mathrm{cfu} / \mathrm{g}$ ) had markedly lower $(\mathrm{P}<0.05) \mathrm{PBC}$ other than the other treated groups (untreated (control) $7.08 \pm 6.34,1 \%$ chitosan $6.99 \pm 6.08$, and $1 \%$ nanochitosan $6.98 \pm 5.88 \log 10 \mathrm{cfu} / \mathrm{g}$ ) and also markedly lower LBC $(\mathrm{P}<0.05)$ with $2 \%$ chitosan and $2 \%$ nanochitosan treatment groups $(5.88 \pm 5.23$ and $5.58 \pm 5.23 \log 10 \mathrm{cfu} / \mathrm{g})$ than the other 
treated groups (control: $6.79 \pm 6.18,1 \%$ chitosan: $6.70 \pm 6.43$, and $1 \%$ nanochitosan: $6.65 \pm 6.04 \log 10$ cfu/g). Finally, at fifteenth day PBC mean values recorded $8.52 \pm 8.11,7.36 \pm 6.89,6.34 \pm 6.04,7.32 \pm 7.11$, and $6.23 \pm 5.81$ while LBC were $7.69 \pm 6.72,7.15 \pm 7.45,6.51 \pm 6.26,7.1 \pm 7.34$, and $6.39 \pm 5.92 \log 10 \mathrm{cfu} / \mathrm{g}$ in control, $1 \%$ chitosan, $2 \%$ chitosan, $1 \%$ nanochitosan and $2 \%$ nanochitosan group, respectively.

Table 8 revealed that Staphylococcus aureus count in fish samples immersed in 2\% nanochitosan coating was negative in all storage period. Initial Staphylococcus aureus count was 3.64 $3.30,3.5 \pm 3.0,3.0 \pm 2.85,3.34 \pm 3.49 \log 10$ $\mathrm{cfu} / \mathrm{g}$, and zero in control, $1 \%$ chitosan, $2 \%$ chitosan, $1 \%$ nanochitosan and $2 \%$ nanochitosan group, respectively. At fifth day Staphylococcus aureus count reached 5.77 $\pm 5.11,5.45 \pm 4.58,4.72 \pm 4.26,5.32 \pm 4.72 \log 10 \mathrm{cfu} / \mathrm{g}$, and zero; Then increased to reach $6.91 \pm 5.92,6.84 \pm 6.18,5.38 \pm 5.11$, and $6.8 \pm 6.15$ in control, $1 \%$ chitosan, $2 \%$ chitosan, and $1 \%$ nanochitosan group, respectively at fifteenth day.

Table 2. Statistical analysis results of $\mathrm{pH}$ values of fish fillet samples

\begin{tabular}{|c|c|c|c|c|c|}
\hline Groups & Control & $\begin{array}{c}\text { Chitosan } \\
(1 \mathrm{~g} \mathrm{Ch} / 100 \mathrm{ml} \text { DW) }\end{array}$ & $\begin{array}{c}\text { Chitosan } \\
(2 \mathrm{~g} \mathrm{Ch} / 100 \mathrm{ml} \mathrm{DW})\end{array}$ & $\begin{array}{c}\text { Nanochitosan } \\
(1 \mathrm{~g} \text { NCh/100ml DW) }\end{array}$ & $\begin{array}{c}\text { Nanochitosan } \\
(2 \mathrm{~g} \mathrm{NCh} / 100 \mathrm{ml} \text { DW })\end{array}$ \\
\hline First day & $6.18 \pm 0.15^{a}$ & $6.18 \pm 0.16^{\mathrm{a}}$ & $6.09 \pm 0.12^{\mathrm{a}}$ & $6.13 \pm 0.01^{a}$ & $5.96 \pm 0.07^{a}$ \\
\hline $5^{\text {th }}$ day & $6.72 \pm 0.26^{\mathrm{a}}$ & $6.58 \pm 0.02^{\mathrm{ab}}$ & $6.29 \pm 0.01^{b}$ & $6.39 \pm 0.02^{a b}$ & $6.21 \pm 0.01^{b}$ \\
\hline $10^{\text {th }}$ day & $8.22 \pm 0.05^{\mathrm{a}}$ & $6.87 \pm 0.05^{b}$ & $6.30 \pm 0.02^{\mathrm{cd}}$ & $6.46 \pm 0.02^{c}$ & $6.24 \pm 0.03^{\mathrm{d}}$ \\
\hline $15^{\text {th }}$ day & $8.63 \pm 0.06^{\mathrm{a}}$ & $6.88 \pm 0.04^{b}$ & $6.48 \pm 0.02^{\mathrm{d}}$ & $6.62 \pm 0.02^{c}$ & $6.30 \pm 0.02^{\mathrm{e}}$ \\
\hline
\end{tabular}

Means of different superscript letters within the same row differ significantly at P< 0.05. Ch: Chitosan, NCh: Nanochitosan, DW: Distilled water

Table 3. Statistical analysis results of TVB-N values of fish fillet samples

\begin{tabular}{lccccc}
\hline Groups & Control & $\begin{array}{c}\text { Chitosan } \\
(\mathbf{1 g ~ C h} / \mathbf{1 0 0 m l} \text { DW })\end{array}$ & $\begin{array}{c}\text { Chitosan } \\
(\mathbf{2 g ~ C h} / \mathbf{1 0 0 m l} \mathbf{D W})\end{array}$ & $\begin{array}{c}\text { Nanochitosan } \\
(\mathbf{1 g ~ N C h} / \mathbf{1 0 0 m l} \mathbf{D W})\end{array}$ & $\begin{array}{c}\text { Nanochitosan } \\
(\mathbf{2 g} \text { NCh/100ml DW })\end{array}$ \\
\hline First day & $6.18 \pm 1.24^{\mathrm{a}}$ & $6.03 \pm 1.18^{\mathrm{a}}$ & $5.04 \pm 0.83^{\mathrm{a}}$ & $6.04 \pm 1.22^{\mathrm{a}}$ & $4.89 \pm 0.73^{\mathrm{a}}$ \\
$5^{\text {th }}$ day & $36.73 \pm 0.26^{\mathrm{a}}$ & $22.03 \pm 0.1^{\mathrm{b}}$ & $16.73 \pm 0.34^{\mathrm{d}}$ & $19.15 \pm 0.13^{\mathrm{c}}$ & $14.01 \pm 1.1^{\mathrm{e}}$ \\
$10^{\text {th }}$ day & $47.88 \pm 0.79^{\mathrm{a}}$ & $29.99 \pm 0.63^{\mathrm{b}}$ & $20.68 \pm 0.9^{\mathrm{c}}$ & $27.04 \pm 1.32^{\mathrm{b}}$ & $17.95 \pm 0.53^{\mathrm{c}}$ \\
$15^{\text {th }}$ day & $54.62 \pm 0.27^{\mathrm{a}}$ & $35.92 \pm 0.20^{\mathrm{b}}$ & $22.93 \pm 0.55^{\mathrm{c}}$ & $34.64 \pm 0.9^{\mathrm{b}}$ & $19.75 \pm 0.93^{\mathrm{d}}$ \\
\hline
\end{tabular}

Means of different superscript letters within the same row differ significantly at $\mathrm{P}<0.05$. Ch: Chitosan, NCh: Nanochitosan, DW: Distilled water

Table 4. Statistical analysis results of TBA values of fish fillet samples

\begin{tabular}{lccccc}
\hline Groups & Control & $\begin{array}{c}\text { Chitosan } \\
(\mathbf{1 g ~ C h} / \mathbf{1 0 0 m l} \mathbf{D W})\end{array}$ & $\begin{array}{c}\text { Chitosan } \\
(\mathbf{2 g} \text { Ch/100ml DW })\end{array}$ & $\begin{array}{c}\text { Nanochitosan } \\
(\mathbf{1 g ~ N C h} / \mathbf{1 0 0 m l} \mathbf{D W})\end{array}$ & $\begin{array}{c}\text { Nanochitosan } \\
(\mathbf{2 g} \text { NCh/100ml DW })\end{array}$ \\
\hline First day & $1.26 \pm 0.45^{\mathrm{a}}$ & $1.12 \pm 0.40^{\mathrm{a}}$ & $0.67 \pm 0.19^{\mathrm{a}}$ & $0.90 \pm 0.04^{\mathrm{a}}$ & $0.56 \pm 0.15^{\mathrm{a}}$ \\
$5^{\text {th }}$ day & $4.83 \pm 0.06^{\mathrm{a}}$ & $3.89 \pm 0.07^{\mathrm{b}}$ & $2.24 \pm 0.04^{\mathrm{c}}$ & $3.23 \pm 0.29^{\mathrm{b}}$ & $1.18 \pm 0.24^{\mathrm{c}}$ \\
$10^{\text {th }}$ day & $5.60 \pm 0.04^{\mathrm{a}}$ & $4.69 \pm 0.05^{\mathrm{b}}$ & $3.55 \pm 0.20^{\mathrm{d}}$ & $4.24 \pm 0.06^{\mathrm{c}}$ & $3.21 \pm 0.04^{\mathrm{e}}$ \\
$15^{\text {th }}$ day & $6.87 \pm 0.03^{\mathrm{a}}$ & $4.78 \pm 0.06^{\mathrm{b}}$ & $4.16 \pm 0.09^{\mathrm{cd}}$ & $4.31 \pm 0.04^{\mathrm{c}}$ & $3.95 \pm 0.05^{\mathrm{d}}$ \\
\hline
\end{tabular}

Means of different superscript letters within the same row differ significantly at $\mathrm{P}<0.05$. Ch: Chitosan, NCh: Nanochitosan, DW: Distilled water

Table 5. Statistical analysis results of Total bacterial count (TBC) of fish fillet samples

\begin{tabular}{lccccc}
\hline Groups & Control & $\begin{array}{c}\text { Chitosan } \\
(\mathbf{1 g} \text { Ch/100ml DW })\end{array}$ & $\begin{array}{c}\text { Chitosan } \\
(\mathbf{2 g} \text { Ch/100ml DW })\end{array}$ & $\begin{array}{c}\text { Nanochitosan } \\
(\mathbf{1 g ~ N C h} / \mathbf{1 0 0 m l} \mathbf{D W})\end{array}$ & $\begin{array}{c}\text { Nanochitosan } \\
(\mathbf{2 g} \mathbf{g} \mathbf{~ N h} / \mathbf{1 0 0 m l} \mathbf{D W})\end{array}$ \\
\hline First day & $5.15 \pm 4.23^{\mathrm{a}}$ & $5.0 \pm 4.64^{\mathrm{a}}$ & $4.18 \pm 3.54^{\mathrm{b}}$ & $5.04 \pm 4.58^{\mathrm{a}}$ & $3.97 \pm 3.36^{\mathrm{b}}$ \\
$5^{\text {th }}$ day & $5.92 \pm 5.41^{\mathrm{a}}$ & $5.11 \pm 4.36^{\mathrm{b}}$ & $4.88 \pm 4.45^{\mathrm{b}}$ & $5.08 \pm 4.36^{\mathrm{b}}$ & $4.95 \pm 3.46^{\mathrm{b}}$ \\
$10^{\text {th }}$ day & $6.26 \pm 5.41^{\mathrm{a}}$ & $6.11 \pm 5.97^{\mathrm{ab}}$ & $5.29 \pm 4.82^{\mathrm{b}}$ & $6.08 \pm 5.62^{\mathrm{ab}}$ & $4.99 \pm 4.51^{\mathrm{b}}$ \\
$15^{\text {th }}$ day & $7.89 \pm 7.72^{\mathrm{a}}$ & $7.71 \pm 7.38^{\mathrm{a}}$ & $6.78 \pm 6.65^{\mathrm{a}}$ & $7.69 \pm 7.34^{\mathrm{a}}$ & $6.28 \pm 6.08^{\mathrm{a}}$ \\
\hline
\end{tabular}

Means of different superscript letters within the same row differ significantly at P < 0.05. Ch: Chitosan, NCh: Nanochitosan, DW: Distilled water 
Table 6. Statistical analysis results of proteolytic bacterial counts of fish fillet samples

\begin{tabular}{lccccc}
\hline Groups & Control & $\begin{array}{c}\text { Chitosan } \\
(\mathbf{1 g ~ C h} / \mathbf{1 0 0 m l} \mathbf{D W})\end{array}$ & $\begin{array}{c}\text { Chitosan } \\
(\mathbf{2 g ~ C h} / \mathbf{1 0 0 m l} \mathbf{D W})\end{array}$ & $\begin{array}{c}\text { Nanochitosan } \\
(\mathbf{1 g ~ N C h} / \mathbf{1 0 0 m l} \mathbf{D W})\end{array}$ & $\begin{array}{c}\text { Nanochitosan } \\
(\mathbf{2 g} \mathbf{\text { NCh}} / \mathbf{1 0 0 m l} \mathbf{D W})\end{array}$ \\
\hline First day & $4.57 \pm 2.95^{\mathrm{a}}$ & $4.41 \pm 2.95^{\mathrm{a}}$ & $4.08 \pm 3.18^{\mathrm{a}}$ & $4.11 \pm 4.41^{\mathrm{a}}$ & $4.04 \pm 3.98^{\mathrm{a}}$ \\
$5^{\text {th }}$ day & $5.23 \pm 3.76^{\mathrm{a}}$ & $5.11 \pm 4.18^{\mathrm{ab}}$ & $5.04 \pm 4.08^{\mathrm{b}}$ & $5.08 \pm 3.94^{\mathrm{b}}$ & $4.72 \pm 4.32^{\mathrm{c}}$ \\
$10^{\text {th }}$ day & $7.08 \pm 6.34^{\mathrm{a}}$ & $6.99 \pm 6.08^{\mathrm{a}}$ & $5.85 \pm 5.28^{\mathrm{b}}$ & $6.98 \pm 5.88^{\mathrm{a}}$ & $5.71 \pm 5.41^{\mathrm{b}}$ \\
$15^{\text {th }}$ day & $8.52 \pm 8.11^{\mathrm{a}}$ & $7.36 \pm 6.89^{\mathrm{b}}$ & $6.34 \pm 6.04^{\mathrm{b}}$ & $7.32 \pm 7.11^{\mathrm{b}}$ & $6.23 \pm 5.81^{\mathrm{b}}$ \\
\hline
\end{tabular}

Means of different superscript letters within the same row differ significantly at $\mathrm{P}<0.05$. Ch: Chitosan, NCh: Nanochitosan, DW: Distilled water

Table 7. Statistical analysis results of lipolytic bacterial counts of fish fillet samples

\begin{tabular}{lccccc}
\hline Groups & Control & $\begin{array}{c}\text { Chitosan } \\
(\mathbf{1 g ~ C h} / \mathbf{1 0 0 m l} \mathbf{D W})\end{array}$ & $\begin{array}{c}\text { Chitosan } \\
(\mathbf{2 g ~ C h} / \mathbf{1 0 0 m l} \mathbf{D W})\end{array}$ & $\begin{array}{c}\text { Nanochitosan } \\
(\mathbf{1 g ~ N C h} / \mathbf{1 0 0 m l} \mathbf{D W})\end{array}$ & $\begin{array}{c}\text { Nanochitosan } \\
(\mathbf{2 g ~ N C h} / \mathbf{1 0 0 m l ~ D W})\end{array}$ \\
\hline First day & $3.98 \pm 3.89^{\mathrm{a}}$ & $3.71 \pm 3.71^{\mathrm{a}}$ & $3.28 \pm 3.60^{\mathrm{a}}$ & $3.68 \pm 4.28^{\mathrm{a}}$ & - \\
$5^{\text {th }}$ day & $5.46 \pm 4.26^{\mathrm{a}}$ & $5.36 \pm 4.58^{\mathrm{a}}$ & $4.71 \pm 4.53^{\mathrm{a}}$ & $5.3 \pm 5.32^{\mathrm{a}}$ & $4.51 \pm 4.04^{\mathrm{a}}$ \\
$10^{\text {th }}$ day & $6.79 \pm 6.18^{\mathrm{a}}$ & $6.70 \pm 6.43^{\mathrm{a}}$ & $5.88 \pm 5.23^{\mathrm{b}}$ & $6.65 \pm 6.04^{\mathrm{a}}$ & $5.58 \pm 5.23^{\mathrm{b}}$ \\
$15^{\text {th }}$ day & $7.69 \pm 6.72^{\mathrm{a}}$ & $7.15 \pm 7.45^{\mathrm{a}}$ & $6.51 \pm 6.26^{\mathrm{a}}$ & $7.1 \pm 7.34^{\mathrm{a}}$ & $6.39 \pm 5.92^{\mathrm{a}}$ \\
\hline
\end{tabular}

Means of different superscript letters within the same row differ significantly at $\mathrm{P}<0.05$. Ch: Chitosan, NCh: Nanochitosan, DW: Distilled water

Table 8. Statistical analysis results of Staphylococcus aureus count of fish fillet samples

\begin{tabular}{lccccc}
\hline Groups & Control & $\begin{array}{c}\text { Chitosan } \\
(\mathbf{1 g} \text { Ch/100ml DW })\end{array}$ & $\begin{array}{c}\text { Chitosan } \\
(\mathbf{2 g} \text { Ch/100ml DW })\end{array}$ & $\begin{array}{c}\text { Nanochitosan } \\
(\mathbf{1 g} \text { NCh/100ml DW })\end{array}$ & $\begin{array}{c}\text { Nanochitosan } \\
(\mathbf{2 g} \mathbf{~ N C h} / \mathbf{1 0 0 m l} \mathbf{D W})\end{array}$ \\
\hline First day & $3.64 \pm 3.30^{\mathrm{a}}$ & $3.5 \pm 3.0^{\mathrm{a}}$ & $3.0 \pm 2.85^{\mathrm{a}}$ & $3.34 \pm 3.49^{\mathrm{a}}$ & - \\
$5^{\text {th }}$ day & $5.77 \pm 5.11^{\mathrm{a}}$ & $5.45 \pm 4.58^{\mathrm{ab}}$ & $4.72 \pm 4.26^{\mathrm{b}}$ & $5.32 \pm 4.72^{\mathrm{b}}$ & - \\
$10^{\text {th }}$ day & $6.76 \pm 6.32^{\mathrm{a}}$ & $6.72 \pm 6.75^{\mathrm{a}}$ & $5.82 \pm 4.83^{\mathrm{a}}$ & $6.61 \pm 6.15^{\mathrm{a}}$ & - \\
$15^{\text {th }}$ day & $6.91 \pm 5.92^{\mathrm{a}}$ & $6.84 \pm 6.18^{\mathrm{a}}$ & $5.38 \pm 5.11^{\mathrm{b}}$ & $6.8 \pm 6.15^{\mathrm{a}}$ & - \\
\hline
\end{tabular}

Means of different superscript letters within the same row differ significantly at $\mathrm{P}<0.05$. Ch: Chitosan, NCh: Nanochitosan, DW: Distilled water

\section{DISCUSSION}

\section{Sensory evaluation}

Fish acceptability and its products through storing relied on the variations in their sensory characteristics. Fish fillets were deemed to be satisfactory for human consuming until the sensory grade reached 4 (Ojagh et al., 2010). Among treatments, the most elevated score was gotten for the fish slices coated with $2 \%$ nanochitosan.

\section{Physicochemical analysis}

pH

$2 \%$ Chitosan and $2 \%$ nanochitosan groups were remarkably lower in $\mathrm{pH}$ values than the other sets $(\mathrm{P}<0.05)$ due to the suppression in development of bacteria (Shahidi, et al., 1999) and were acceptable according to EOS (2005) where $\mathrm{pH}$ of fish meat shouldn't exceed 6.5. The gradual increase of $\mathrm{pH}$ rates in refrigerating storing periods, probably due to the collection of fundamental components created from both autolysis handled by endogenous enzymes and microbial enzymatic activities (Nirmal and Benjakul, 2011). Similar observations were made by Alboghbeish and Khodanazary (2018). The $\mathrm{pH}$ is a substantial determinant of microbial development and seafood with elevated $\mathrm{pH}$ has a high spoilage possibility and a brief shelf life (Newton and Gell, 1981).

\section{Total volatile basic nitrogen}

The Total volatile basic nitrogen (TVB-N) value is a pointer of spoilage, which is basically consisted of trimethylamine, dimethylamine, and ammonia resulted from the degeneration of proteins and non-protein nitrogenous components by the action of spoilage microbes and endogenous enzymes. It was noticed that the rate of TVB-N rising was extremely slower in fish slices coated with chitosan and nanochitosan rather than the control samples. Besides, a significant difference $(\mathrm{P}<0.05)$ was in TVB-N values between $1 \%$ and $2 \%$ chitosan, and also, among $1 \%$ and $2 \%$ nanochitosan treated groups on days 5, 10, and 15. Rates of TVB-N in groups of $1 \%$ chitosan and nanochitosan were higher than $2 \%$ chitosan and nanochitosan groups, this might be ascribed to the higher antimicrobial action of $2 \%$ chitosan and nanochitosan compared to $1 \%$ chitosan and nanochitosan. TVB-N mean values of $2 \%$ chitosan and $2 \%$ nanochitosan at fifteenth day of storage were acceptable according to EOS (2005) rather than the other groups where 
TVB-N of fish meat should be 30mg/100g. Ramezani et al. (2015) and Ojagh et al. (2010) revealed that pretreatment of silver carp and rainbow trout with $2 \%$ nanochitosan and $2 \%$ chitosan respectively, might delay the rising in the TVB-N rates compared to the other treated groups. Also, Fan et al. (2009) explained that chitosan coating decreased TVB-N values obviously and consequently slowed the deterioration of silver carp.

\section{Thiobarbituric acid reactive substances}

Thiobarbituric acid reactive substances value has been commonly used to measure the grade of lipid oxidation and the existence of TBA reactive substances is attributable to the second step auto-oxidation through which aldehyde and ketone are produced from peroxides oxidation. During the storage period, samples coated with $2 \%$ chitosan and nanochitosan had significantly lower TBA values than samples coated with $1 \%$ chitosan and nanochitosan $(\mathrm{P}<0.05)$. At day 15 of storing, TBA mean values of $2 \%$ chitosan and 1,2\% nanochitosan were acceptable according to EOS (2005) rather than control and $1 \%$ chitosan where TBA exceeded $4.5 \mathrm{MDA} / \mathrm{kg}$ of examined samples. The raising in TBA levels of samples through storing can be ascribed to the partial dehydration of fish and interaction of lipids with the oxygen of air (Kilincceker et al., 2009). Therefore, the use of chitosan coating possibly will reduce lipid oxidation in fish samples due to the antioxidant activity and its low oxygen permeability characteristic. Solval et al. (2014) confirmed that the coating of chitosan nanoparticles through frozen storage could reduce the TBARS content in the shrimp. Also, Alboghbeish and Khodanazary (2018) reported that 2\% chitosan and nanochitosan may minimize lipid oxidation levels in Carangoides coeruleopinnatus fillets during refrigerated storage.

\section{Microbiological analysis}

It is noteworthy that TBC of fish fillets in the control group raised quickly during the storage period and was significantly higher than the other treated groups $(\mathrm{P}<0.05)$, demonstrating the antimicrobial action of chitosan and chitosan nanoparticles and exceeded the maximum acceptability level of EOS $\left(2005,10^{6} \mathrm{cfu} / \mathrm{g}\right)$ on day 10 . Therefore, the treatment of fish coated with $2 \%$ chitosan and $2 \%$ nanochitosan might delay the development of total bacteria more efficiently, compared with $1 \%$ chitosan and $1 \%$ nanochitosan. The mechanism of action of chitosan seems to be related to the disruption of the lipopolysaccharide layer of outer membrane of Gram-negative bacteria (Pereda et al., 2011), as well as to its role as a buffer against oxygen transfer (Jeon et al., 2002).Seafood spoiled by proteolytic and lipolytic bacterial strains which are capable of producing extracellular protease and lipase enzymes that can break down protein and fat to substances with low molecular weight. Protease enzymes can target the nitrogen molecules that occur naturally in meat, causing severe deteriorating color and odor changes in foods even when preserved in refrigeration or frozen (Ali, 2011). Chitosan and nanochitosan treated samples showed a decrease in PBC and LBC values compared to control samples that suggest the antimicrobial activity of chitosan and chitosan nanoparticles. The groups treated with $2 \%$ chitosan and 2\% nanochitosan had significantly lower PBC and LBC $(\mathrm{P}<0.05)$ than the other treated groups at tenth day of the storage period. Proteolytic and lipolytic bacteria could be responsible for a variety of food odor and flavor problems. Some of the common psychrotrophic bacteria are intensely proteolytic and/or lipolytic and cause severe defects in dairy, meat, poultry and fish products when high counts $\left(10^{6}\right.$ per g or $\mathrm{ml}$ or higher $)$ are reached during chilled storage (Vanderzant et al., 1985). The results confirmed the antibacterial properties of nanochitosan as stated by Ramezani et al. (2015). Dipping of samples in this solution prevented oxidation of flesh and water absorption and thus inhibited bacterial growth, as Fan et al. (2009) observed when investigating the effect of chitosan coating on the quality of silver carp and shelf life during frozen storage. In addition, nanochitosan solution is known to degrade bacterial cell walls naturally, rendering them vulnerable to lysis, which has resulted in lethal consequences (Liu et al., 2004). Fish samples immersed in 2\% nanochitosan coating were negative for Staphylococcus aureus in all storage periods. During the storage period, there is a decrease in Staphylococcus aureus count of 2\% chitosan group than the other treated groups and control samples $(\mathrm{P}<0.05)$. Qi et al. (2004) stated the higher antibacterial activity of chitosan nanoparticles against Escherichia coli, Staphylococcus aureus, and Salmonella typhimurium than chitosan due to the extraordinary nature of the nanoparticles, the nanoparticles are probable to have a larger surface area and a greater affinity with the microbial cells, producing a quantum-sized effect. On the contrary, Sadeghi et al. (2008) mentioned that the impact of chitosan nanoparticles on Staphylococcus aureus is less inhibitive than free-soluble polymers, where nanoparticles have less positive binding charges available to link to the negative bacterial cell wall. On the other hand, Du et al. (2009) stated that nanoparticles of chitosan tripolyphosphate loaded with different metal ions display greater antibacterial activity against Escherichia coli, Salmonella choleraesuis, and Staphylococcus aureus.

\section{CONCLUSION}

Chitosan is a potential resource that is sustainable, non-toxic and biodegradable and has gained significant attention in the final two decades. Results of this study displayed a shelf-life of fewer than 5 days for untreated tilapia (Oreochromis niloticus) slices, while a shelf-life of 10 days was observed for $2 \%$ chitosan and $2 \%$ nanochitosan treated samples according to various quality and spoilage parameters, where the bacterial and chemical examination was associated with 
the sensory assessment. In addition, $2 \%$ nanochitosan demonstrated a greater ability to inhibit TVB-N and TBARS content compared to other treated groups, resulting in delaying the deterioration of fresh tilapia slices and prolonged shelf life during chilled storage. So, our work demonstrated the antioxidant and antimicrobial activity of chitosan and nanochitosan as a natural preservative for preserving of tilapia fillets during refrigerated storage.

\section{DECLARATIONS}

\section{Acknowledgements}

Great thanks are extended to all staff members in Animal Health Research Institute, department of Food Hygiene, Egypt.

\section{Competing interests}

The authors declared that they have no competing interests.

\section{Consent to publish}

All authors agree on article publication

\section{Author`s contributions}

WME and SAY found research idea, planned the study design, performed data, performed the laboratory work, collected of fish samples in the experiment, drafted and preparation the manuscript. MNS shared in the research's idea and preparation of working solutions. The final manuscript was read and accepted by all authors.

\section{REFERENCES}

Alboghbeish H and Khodanazary A (2018). The Comparison of Quality Characteristics of Refrigerated Carangoides coeruleopinnatus Fillets with Chitosan and Nanochitosan Coating. Turkish. Journal of Fisheries\& Aquatic Sciences, 19: 957-967. DOI: https://doi.org/10.4194/1303-2712v19_11_07

American Public Health Association (APHA) (2002). Compendium of Methods for the Microbial Examination of Food, 3rd Ed., American Public Health Association, Washington DC 20005 USA, pp. 183-198. DOI: https://doi.org/10.2105/MBEF.0222sec1

Cuero RG, Osuji G and Washington ANC (1991). N-Carboxymethyl chitosan inhibition of aflatoxin production: role of zinc. Biotechnology Letters Journal, 13(6): 441-444. DOI: https://doi.org/10.1007/BF01030998

Decker EA and Haultin HO (1992). Lipid oxidation in muscle foods via redox ion. In Allen J. Angelo (Ed.), Lipid oxidation in food, pp. 33-54. Washington, D.C.: American Chemical Society. DOI: https://doi.org/10.1021/bk-1992-0500.ch003

Du WL, Niu SS, Xu YL, Xu ZR and Fan CL (2009). Antibacterial activity of chitosan tripolyphosphate nanoparticles loaded with various metal ions. Carbohydrate Polymers journal, 75: 385-389. DOI: https://doi.org/10.1016/j.carbpol.2008.07.039

Egyptian Organization for Standardization and Quality Control (ESO) (2005). Chilled fish, (Standard number: 3494). Available at: http://www.eos.org.eg

Egyptian Organization for Standardization (ES) (2006). Methods of analysis and testing for meat. Part 9: determination of total volatile nitrogen (TVN). (Standard number: 63-9/2006). Available at: http://www.eos.org.eg

Egyptian Organization for Standardization (ES) (2006). Methods of analysis and testing for meat. Part 10: determination of thiobarbituric acid (TBA). (Standard number: 63-10/2006). Available at: http://www.eos.org.eg

Fan WJ, Sun JX, Chen YC, Qiu J, Zhang Y and Chi YL (2009). Effects of chitosan coating on quality and shelf life of silver carp during frozen storage. Food Chemistry journal, 115: 66-70. DOI: https://doi.org/10.1016/j.foodchem.2008.11.060

Fang SW, Li CF and Shih DYC (1994). Antifungal activity of chitosan and its preservative effect on low-sugar candied kumquat. Journal of food protection 57:136-140. DOI:https://doi.org/10.4315/0362-028X-57.2.136

Ali HM Fatma (2011). Quality Evaluation of Some Fresh and Imported Frozen Seafood. Advance Journal of Food Science and Technology 3: 8388.DOI: https://doi.org/10.12691/ajfst-1-4-3

Friedman M and Juneja VK (2010). Review of antimicrobial and antioxidative activities of chitosans in food. Journal of Food Protection, 73 (9): 17371761. DOI: https://doi.org/10.4315/0362-028x-73.9.1737

Jeon CO, Kamil YVA and Shahidi F (2002). Chitosan as an edible invisible film for quality preservation of herring and Atlantic cod. Journal of Agricultural and Food Chemistry, 50: 5167-5178. DOI: https://doi.org/10.1021/jf0116931

Kilincceker O, Dogan IS and Kucukoner E (2009). Effect of edible coatings on the quality of frozen fish fillets. LWT -Journal of Food Science and Technology, 42: 868-873. DOI: https://doi.org/10.1016/j.lwt.2008.11.003

Kong M, Chen XG, Xing K and Park HJ (2010). Antimicrobial properties of chitosan and mode of action: a state of the art review. International Journal of Food Microbiology, 144: 51-63. DOI: https://doi.org/10.1016/j.ijfoodmicro.2010.09.012

Liu H, Du Y, Wang X and Sun L (2004). Chitosan kills bacteria through cell membrane damage. International journal of food microbiology, 95: 147155. DOI: https://doi.org/10.1016/j.ijfoodmicro.2004.01.022

Newton KG and Gell CO (1981). The microbiology of dark firm and dry fresh meats: A Review. Journal of Meat Science, 95: 223-232. DOI: https://doi.org/10.1016/0309-1740(81)90005-X

Nirmal NP and Benjakul S (2011). Retardation of quality changes of Pacific white shrimp by green tea extract treatment and modified atmosphere packaging during refrigerated storage. International Journal of Food Microbiology, 149: 247-253. DOI: https://doi.org/10.1016/j.ijfoodmicro.2011.07.002

Ojagh SM, Rezaei M, Razavi SH and Hosseini SMH (2010). Effect of chitosan coatings enriched with cinnamon oil on the quality of refrigerated rainbow trout. Food Chemistry Journal, 120: 193-198. DOI: https://doi.org/ 10.1016/j.foodchem.2009.10.006 
Papineau AM, Hoover DG, Knorr D and Farkas DF (1991). Antimicrobial effect of water soluble chitosans with high hydrostatic pressure. Food Biotechnology Journal, 5:45-57. DOI:https://doi.org/10.1080/08905439109549790

Pearson D (2006). Chemical Analysis of Foods. $11^{\text {th }}$ Ed, Publishing Co., Churchill Livingstone, Edinburgh, London, United Kingdom. Available at: https://trove.nla.gov.au/version/45250339

Pereda M, Ponce AG, Marcovich NE, Ruseckaite RA and Martucci JF (2011). Chitosan-gelatin composites and bi-layer films with potential antimicrobial activity. Food Hydrocolloids Journal, 25: 1372-1381. DOI: https://doi.org/10.1016/j.foodhyd.2011.01.001

Qi LF, Xu ZR, Jiang X, Hu CH and Zou XF (2004). Preparation and antibacterial activity of chitosan nanoparticles. Carbohydrate Research Journal, 339: 2693-2700. DOI: https://doi.org/10.1016/j.carres.2004.09.007

Raafat D and Sahl HG (2009). Chitosan and its antimicrobial potential - a critical literature survey. Journal of Microbial Biotechnology, 2(2): 186-201. DOI: https://doi.org/10.1111/j.1751-7915.2008.00080.x

Ramezani Z, Zarei M and Raminnejad N (2015). Comparing the effectiveness of chitosan and nanochitosan coatings on the quality of refrigerated silver carp fillets. Food Control Journal, 51: 43-48. DOI: https://doi.org/10.1016/j.foodcont.2014.11.015

Sadeghi AMM, Dorkoosh FA, Avadi MR, Saadat P, Rafiee-Tehrani M and Junginger HE (2008). Preparation, characterization and antibacterial activities of chitosan, N-trimethyl chitosan (TMC) and N-diethylmethyl chitosan (DEMC) nanoparticles loaded with insulin using both the ionotropic gelation and polyelectrolyte complexation methods. International Journal of Pharmaceutics, 355: 299-306. DOI: https://doi.org/10.1016/j.ijpharm.2007.11.052

Shahidi F, Kamil J, Arachchi V and Jeon YJ (1999). Food applications of chitin and chitosans. Trends in Food Science and Technology Journal, 10: 37-51. DOI: https://doi.org/10.1016/S0924-2244(99)00017-5

Sinha ray S and Okamoto M (2003). Polymer layered silicate nanocomposites. A review from preparation to processing. Progress in polymer Science journal, 28:1539. DOI: https://doi.org/10.1016/j.progpolymsci.2003.08.002

Solval KM, Espinoza Rodezno LA, Moncada M, Bankston JD and Sathivel S (2014). Evaluation ofchitosan nanoparticles as a glazing material for cryogenically frozen shrimp. LWT- Journal of Food Science and Technology, 57: 172-180. DOI: https://doi.org/10.1016/j.1wt.2013.12.033

Sudarshan NR, Hoover DG and Knorr D (1992). Antibacterial action of chitosan. Food Biotechnology Journal, 6: 257-272. DOI: https://doi.org/10.1080/08905439209549838

VanderzantC.,Splittstoesser FD, Ashton HD, Bryan LF,Collins-Thompson LD, FosterME,JezeskiJJ,LechowichVR, Marino JR, Olson CJ et al. (1985). An evaluation of the role of microbiological criteria for foods and food ingredients. National Research Council (US) Subcommittee on Microbiological Criteria. Washington (DC): National Academies Press (US). DOI: $\underline{\text { https://doi.org/10.17226/372 }}$

Zhang Y, Yang Y, Tang K, Hu X and Zou G (2008). Physicochemical characterization and antioxidant activity of quercetin-loaded chitosan nanoparticles. Journal of Applied Polymer Science, 107: 891-897. DOI: https://doi.org/10.1002/app.26402 